\title{
Expansions for the Dirac Operator and Related Operators in Super Spinor Space
}

\author{
Hongfen Yuan
}

\begin{abstract}
In this paper, we study expansions for the Dirac operator $D$, the modified Dirac operator $D-\lambda$, and the polynomial Dirac operator $P(D)$ in super spinor space. These expansions are a meaningful generalization of the classical Almansi expansion in polyharmonic functions theory. As an application of the expansions, the generalized Riquier problem in super spinor space is investigated.
\end{abstract}

Keywords. Super spinor space, Polynomial Dirac operator, Almansi expansion, Riquier problem.

\section{Introduction}

In 2013, Coulembier constructed the spinor representation for the orthosymplectic superalgebra osp $(m \mid 2 n), \mathbb{S}_{m \mid 2 n}$ (see [5]), which generalizes the $\mathfrak{s o}(m)$ spinors (see [9]) and the symplectic spinors for $\mathfrak{s p}(2 n)$ (see [12]). Furthermore, he studied the complete decomposition of a certain class of tensor product representations for $\mathfrak{o s p}(m \mid 2 n)$. In [7], Coulembier and De Bie defined the Dirac operator, acting on super functions defined on $\mathbb{R}^{m \mid 2 n}$ with values in super spinor space $\mathbb{S}_{m \mid 2 n}$, which generalizes the Cauchy-Riemann operators by Stein and Weiss (see [19]). The Dirac operator is the natural extension of both the classical Dirac operator, for the case $n=0$, which acts on the functions defined $\mathbb{R}^{m}$ with values in the orthogonal spinors $\mathbb{S}_{m}$ (see [8]), and the symplectic Dirac operator, for the case $m=0$, which acts on $\mathfrak{s p}(2 n)$ on differential forms on $\mathbb{R}^{2 n}$ with values in the symplectic spinors $\mathbb{S}_{0 \mid 2 n}$ (see [13]). Moreover, they defined a Laplace operator in super spinor space and studied Fischer decomposition (that is, arbitrary polynomials can be decomposed into a sum of products of the powers of the vector variable with spherical monogenics). Based on their work, we investigate Almansi type expansions in super spinor space.

In 1899, Almansi [1] proved the following remarkable statement: if $f(x)$ is a polyharmonic function of order $m$ in a star-shaped domain $\Omega$ centered at the origin of coordinates, then there exist single-valued harmonic functions $f_{0}(x), \ldots, f_{m-1}(x)$ in $\Omega$ such that 


$$
f(x)=f_{0}(x)+|x|^{2} f_{1}(x)+\cdots+|x|^{2(m-1)} f_{m-1}(x) .
$$

The expansion is the so-called Almansi expansion. Indeed the expansion builds the relation between harmonic functions and polyharmonic functions, which plays a central role in the study of polyharmonic functions. The result in the case of complex analysis, several complex variables, and Clifford analysis have been well developed in $[3,14,18]$. More recent generalizations of the result, for kernels of iterated differential operators, such as the iterates of weighted Laplace and Helmholtz operators, can be found in $[11,16]$. In addition, the ideas of Almansi expansion is useful in the study of partial differential equations and boundary value problems (see $[2,4,15]$ ). Most recently, we have studied Almansi expansions for the Dirac operator and the Laplace operator in superspace (see $[17,20,21]$ ). But as we know, up to now there is no hint on the Almansi expansion for polynomial Dirac operator in spinor space. We try to fill part of this gap. In this paper, we mainly study expansions for the modified Dirac operator $D-\lambda$, and the polynomial Dirac operator $P(D)$ in super spinor space. Furthermore, we investigate the generalized Riquier problem in super spinor space by the expansion for the operator $D-\lambda$.

\section{Preliminaries}

\section{1. $\mathbb{Z}_{\mathbf{2}}$-Graded Algebra}

The flat supermanifold, which contains $m$ commuting (bosonic) and $2 n$ anticommuting (fermionic) co-ordinates, is denoted by $\mathbb{R}^{m \mid 2 n}$. The superalgebra $\left(\mathbb{Z}_{2}\right.$-graded algebra) of functions on this flat supermanifold $\mathbb{R}^{m \mid 2 n}$ is

$$
O\left(\mathbb{R}^{m \mid 2 n}\right)=C^{\infty}\left(\mathbb{R}^{m}\right) \otimes \Lambda_{2 n}
$$

where $\Lambda_{2 n}$ is the Grassmann algebra generated by $2 n$ anti-commuting variables, denoted by $\grave{x}_{i}$.

The supervector $\mathbf{x}$ is defined to be

$$
\mathbf{x}=\left(X_{1}, \ldots, X_{m+2 n}\right)=(\underline{x}, \underline{\grave{x}})=\left(x_{1}, \ldots, x_{m}, \grave{x}_{1}, \ldots, \grave{x}_{2 n}\right) .
$$

The first $m$ variables are commuting and the last $2 n$ variables are anticommuting. The commutation relations are then summarized in

$$
X_{i} X_{j}=(-1)^{[i][j]} X_{j} X_{i}, \quad i, j=1, \ldots, m+2 n,
$$

where $[i]=0$ if $i=m$ and $[i]=1$ otherwise.

The algebra generated by the variables $X_{j}$ is denoted by $\mathcal{P}$ and is isomorphic to the supersymmetric tensor power of $\mathbb{C}^{m \mid 2 n}$. The partial derivatives are defined by the relation

$$
\partial_{X_{j}} X_{k}=\delta_{j k}+(-1)^{[j][k]} X_{k} \partial_{X_{j}}
$$




\subsection{Super Spinor Space $\mathbb{S}_{m \mid 2 n}$}

The orthosymplectic metric $g \in \mathbb{R}^{(m+2 n) \times(m+2 n)}$ is given in block-matrix form by

$$
g=\left(\begin{array}{ll}
I_{m} & 0 \\
0 & J_{2 n}
\end{array}\right)
$$

with

$$
J_{2 n}=\left(\begin{array}{cc}
0 & I_{n} \\
-I_{n} & 0
\end{array}\right) .
$$

The real orthosymplectic Lie superalgebra $\mathfrak{o s p}(m \mid 2 n)$ can be defined as the subsuperalgebra of $\mathfrak{g l}(m \mid 2 n ; R)$ that preserves this metric.

The spinors $\mathbb{S}_{m \mid 2 n}$ for the orthosymplectic superalgebra osp $(m \mid 2 n)$, as $\mathfrak{o s p}(m \mid 2 n)$-modules, satisfy $\mathbb{S}_{m \mid 2 n} \cong \Lambda_{d \mid n}$, where the complex algebra $\Lambda_{d \mid n}$ is generated by $\left\{\theta_{1}, \ldots, \theta_{d}, t_{1}, \ldots, t_{n}\right\}$ subject to the relations

$$
\theta_{j} \theta_{k}=-\theta_{k} \theta_{j}, \quad 1 \leq j, k \leq d, \quad t_{i} t_{l}=t_{l} t_{i}, \quad 1 \leq i, l \leq n,
$$

and

$$
\theta_{j} t_{i}=-t_{i} \theta_{j}, \quad 1 \leq j \leq d, \quad 1 \leq i \leq n .
$$

This algebra $\Lambda_{d \mid n}$ is a superalgebra with unusual gradation. The commuting variables are considered as odd and the Grassmann variables are even. With this gradation the algebra is in fact a super anti-commutative algebra, $a b=-(-1)^{|a||b|} b a$ for $a, b$ two homogeneous elements of the superalgebra. Therefore this corresponds to a supersymmetric version of a Grassmann algebra.

\subsection{Differential Operators in Super Spinor Space}

The super gradient $\nabla: O\left(\mathbb{R}^{m \mid 2 n}\right) \rightarrow O\left(\mathbb{R}^{m \mid 2 n}\right) \otimes \mathbb{C}^{m \mid 2 n}$ is defined by

$$
\nabla f=\sum_{i=1}^{m+2 n}(-1)^{[i](1+|f|)} \partial_{X_{i}} f \otimes E_{i}, \quad E_{i}=(0, \ldots, 0,1,0, \ldots, 0)
$$

for $O\left(\mathbb{R}^{m \mid 2 n}\right)$ homogeneous.

The super vector space morphism $E^{\perp}: \mathbb{C}^{m \mid 2 n} \otimes \mathbb{S}_{m \mid 2 n} \rightarrow \mathbb{S}_{m \mid 2 n}$ defined by

$$
E^{\perp}\left(E_{k} \otimes v\right)=\sum_{l=1}^{m+2 n}\left(E_{l} \cdot v\right) g_{l k}
$$

is a $\mathfrak{o s p}(m \mid 2 n)$-module morphism.

The Dirac operator in super spinor space $D$ is given by

$$
D f=\partial_{\mathbf{x}} f=E^{\perp}(\nabla f)=\sum_{i, j=1}^{m+2 n} g_{i j} E_{i}\left(\partial_{X_{j}} f\right)
$$

for $f \in O\left(\mathbb{R}^{m \mid 2 n}\right) \otimes \mathbb{S}_{m \mid 2 n}$. 
The null solutions of the super Dirac operator are called super monogenic functions. The null solutions of the operator $D^{k}$ are called k-super monogenic functions.

Besides, we define the Euler operator in super spinor space as

$$
\mathbb{E}=\sum_{i=1}^{m+2 n} X_{i} \partial_{X_{i}}
$$

Note that $\mathbb{E} \mathcal{P}_{k}=k \mathcal{P}_{k}$, where $\mathcal{P}_{k}$ denote the polynomials of degree $k$.

\section{An Expansion for the Operator $D$}

Definition 3.1. We define the generalized super-Euler operator by

$$
\mathbb{U}_{s}=s \mathbf{I}+\mathbb{E}=s \mathbf{I}+\sum_{i=1}^{m+2 n} X_{i} \partial_{X_{i}},
$$

where $s$ is a complex number, $\mathbf{I}$ is the identity operator and $\mathbb{E}$ is the Euler operator in super spinor space.

Lemma 3.2. [7] The operators $\mathbf{x}, \mathbf{x}^{2}, \partial_{\mathbf{x}}, \mathbb{E}$ show the following properties:

$$
\begin{gathered}
\mathbf{x} \partial_{\mathbf{x}}+\partial_{\mathbf{x}} \mathbf{x}=-2 \mathbb{E}-M, \\
\mathbf{x} \mathbb{E}-\mathbb{E} \mathbf{x}=-\mathbf{x}, \\
\partial_{\mathbf{x}} \mathbb{E}-\mathbb{E} \partial_{\mathbf{x}}=\partial_{\mathbf{x}},
\end{gathered}
$$

where $\mathbf{x}=\sum_{i=1}^{m+2 n} X_{i} E_{i}$.

Applying Lemma 3.2, we can obtain the following lemma.

Lemma 3.3. Let $O\left(\mathbb{R}^{m \mid 2 n}\right)=C^{2}\left(\mathbb{R}^{m}\right) \otimes \Lambda_{2 n}$. For $f(\mathbf{x}) \in O\left(\mathbb{R}^{m \mid 2 n}\right) \otimes \mathbb{S}_{m \mid 2 n}$,

$$
\left\{\begin{array}{l}
D\left(\mathbf{x}^{2 s} f(\mathbf{x})\right)=-2 s \mathbf{x}^{2 s-1} f(\mathbf{x})+\mathbf{x}^{2 s} D f(\mathbf{x}) \\
D\left(\mathbf{x}^{2 s-1} f(\mathbf{x})\right)=-2 \mathbf{x}^{2(s-1)} \mathbb{U}_{\frac{M}{2}+s-1} f(\mathbf{x})-\mathbf{x}^{2 s-1} \operatorname{Df}(\mathbf{x}) .
\end{array}\right.
$$

Lemma 3.4. Let $O\left(\Omega^{m \mid 2 n}\right)=C^{2}(\Omega) \otimes \Lambda_{2 n}$. For $f(\mathbf{x}) \in O\left(\Omega^{m \mid 2 n}\right) \otimes \mathbb{S}_{m \mid 2 n}$,

$$
D \mathbb{U}_{s} f(\mathbf{x})=\mathbb{U}_{s+1} D f(\mathbf{x}),
$$

where $s \in \mathbb{C}$.

Proof. Using Definition 3.1 and (3.4), we have the conclusion.

Definition 3.5. An open connected set $\Omega \subset \mathbf{R}^{m}$ is a star domain with center 0 if $\underline{x} \in \Omega$ and $0 \leq t \leq 1$ imply that $t \underline{x} \in \Omega$. The set is denoted by $\Omega_{\star}$.

Definition 3.6. Let $O\left(\Omega_{*}^{m \mid 2 n}\right)=C\left(\Omega_{*}\right) \otimes \Lambda_{2 n}$. The operator $J_{s}$ is defined as

$$
\begin{gathered}
J_{s}: O\left(\Omega_{*}^{m \mid 2 n}\right) \otimes \mathbb{S}_{m \mid 2 n} \rightarrow O\left(\Omega_{*}^{m \mid 2 n}\right) \otimes \mathbb{S}_{m \mid 2 n}, \\
J_{s} f(\mathbf{x})=\int_{0}^{1} f(t \mathbf{x}) t^{s-1} d t
\end{gathered}
$$

where $s>0$. 
Lemma 3.7. Let $O\left(\Omega_{*}^{m \mid 2 n}\right)=C^{1}\left(\Omega_{*}\right) \otimes \Lambda_{2 n}$. For $f(\mathbf{x}) \in O\left(\Omega_{*}^{m \mid 2 n}\right) \otimes \mathbb{S}_{m \mid 2 n}$,

$$
\mathbb{U}_{s} J_{s} f(\mathbf{x})=J_{s} \mathbb{U}_{s} f(\mathbf{x})=f(\mathbf{x}) .
$$

Proof. Using Definitions 3.1 and 3.6, we have

$$
\begin{aligned}
J_{s} \mathbb{U}_{s} f(\mathbf{x}) & =\int_{0}^{1} \mathbb{U}_{s} f(t \mathbf{x}) t^{s-1} d t \\
& =\int_{0}^{1}(s+\mathbb{E}) f(t \mathbf{x}) t^{s-1} d t \\
& =\int_{0}^{1}\left[s f(t \mathbf{x}) t^{s-1}+\sum_{i=1}^{m+2 n} X_{i} \partial_{X_{i}} f(t \mathbf{x}) t^{s-1}\right] d t \\
& =\int_{0}^{1}\left[s f(t \mathbf{x}) t^{s-1}+\sum_{i=1}^{m+2 n} X_{i} \frac{\partial f(t \mathbf{x})}{\partial t X_{i}} t^{s}\right] d t \\
& =\int_{0}^{1} \frac{d}{d t}\left(f(t \mathbf{x}) t^{s}\right) d t \\
& =f(\mathbf{x}) .
\end{aligned}
$$

Similarly, we have

$$
\mathbb{U}_{s} J_{s} f(\mathbf{x})=f(\mathbf{x}) .
$$

Theorem 3.8. Let $O\left(\Omega_{*}^{m \mid 2 n}\right)=C^{k}\left(\Omega_{*}\right) \otimes \Lambda_{2 n}$. If $f(\mathbf{x}) \in O\left(\Omega_{*}^{m \mid 2 n}\right) \otimes \mathbb{S}_{m \mid 2 n}$ satisfies the equation $D^{k} f=0$, then there exist unique super monogenic functions $f_{0}, \cdots, f_{k-1}$ such that

$$
f(\mathbf{x})=f_{0}(\mathbf{x})+\mathbf{x} f_{1}(\mathbf{x})+\mathbf{x}^{2} f_{2}(\mathbf{x})+\cdots+\mathbf{x}^{(k-1)} f_{k-1}(\mathbf{x}),
$$

where

$$
\left\{\begin{array}{l}
f_{k-1}(\mathbf{x})=\frac{(-1)^{k-1}}{2^{k-1}\left[\frac{k-1}{2}\right] !} J_{\frac{M}{2}+\left[\frac{k-1}{2}\right]-1} \cdots J_{\frac{M}{2}} D^{(k-1)} f(\mathbf{x}) \\
f_{k-2}(\mathbf{x})=\frac{(-1)^{k-2}}{2^{k-2}\left[\frac{k-2}{2}\right] !} J_{\frac{M}{2}+\left[\frac{k-2}{2}\right]-1} \cdots J_{\frac{M}{2}} D^{(k-2)}\left[f(\mathbf{x})-\mathbf{x}^{(k-1)} f_{k-1}(\mathbf{x})\right] \\
\vdots \\
f_{1}(\mathbf{x})=\frac{-1}{2} J_{\frac{M}{2}} D\left[f(\mathbf{x})-\mathbf{x}^{2(k-1)} f_{k-1}(\mathbf{x})-\cdots-\mathbf{x}^{2} f_{2}(\mathbf{x})\right] \\
f_{0}(\mathbf{x})=\left[f(\mathbf{x})-\mathbf{x}^{2(k-1)} f_{k-1}(\mathbf{x})-\mathbf{x}^{4} f_{2}(\mathbf{x})-\cdots-\mathbf{x}^{2} f_{1}(\mathbf{x})\right]
\end{array}\right.
$$

Conversely, if functions $f_{0}, \ldots, f_{k-1}$ are super monogenic, then the function $f(\mathbf{x})$ given by (3.7) is k-super monogenic. 
Proof. First we will prove that

$$
D^{l}\left[\mathbf{x}^{l} g(\mathbf{x})\right]=(-1)^{l} 2^{l}\left[\frac{l}{2}\right] ! \mathbb{U}_{\frac{M}{2}} \cdots \mathbb{U}_{\frac{M}{2}+\left[\frac{l}{2}\right]-1} g(\mathbf{x}),
$$

where $g(\mathbf{x}) \in O\left(\Omega_{*}^{m \mid 2 n}\right) \otimes \Lambda_{2 n} \otimes \mathbb{S}_{m \mid 2 n}$ is super monogenic. By Lemma 3.3, for the case $l=2 k$,

$$
\begin{aligned}
D^{2 k}\left[\mathbf{x}^{2 k} g(\mathbf{x})\right] & =D^{(2 k-1)} D\left[\mathbf{x}^{2 k} g(\mathbf{x})\right] \\
& =D^{2(k-1)} D\left[-2 k \mathbf{x}^{2 k-1} g(\mathbf{x})+\mathbf{x}^{2 k} D g(\mathbf{x})\right] \\
& =-2 k D^{2(k-1)}\left[-2 \mathbf{x}^{2(k-1)} \mathbb{U}_{\frac{M}{2}+k-1} g(\mathbf{x})-\mathbf{x}^{2 s-1} D g(\mathbf{x})\right] \\
& =2^{2} k D^{2(k-1)} \mathbf{x}^{2(k-1)} \mathbb{U}_{\frac{M}{2}+k-1} g(\mathbf{x}) \\
& =\cdots \\
& =2^{2 k} k ! \mathbb{U}_{\frac{M}{2}} \cdots \mathbb{U}_{\frac{M}{2}+k-1} g(\mathbf{x}) .
\end{aligned}
$$

For the case $l=2 k-1$,

$$
\begin{aligned}
D^{2 k-1}\left[\mathbf{x}^{2 k-1} g(\mathbf{x})\right] & =D^{2(k-1)} D\left[\mathbf{x}^{2 k-1} g(\mathbf{x})\right] \\
& =D^{2(k-1)}\left[-2 \mathbf{x}^{2(k-1)} \mathbb{U}_{\frac{M}{2}+k-1} g(\mathbf{x})-\mathbf{x}^{2 k-1} D g(\mathbf{x})\right] \\
& =-2 D^{2(k-1)}\left[\mathbf{x}^{2(k-1)} \mathbb{U}_{\frac{M}{2}+k-1} g(\mathbf{x})\right] \\
& =2 \cdot 2^{2(k-1)}(k-1) ! \mathbb{U}_{\frac{M}{2}} \cdots \mathbb{U}_{\frac{M}{2}+k-1} g(\mathbf{x}) .
\end{aligned}
$$

Thus, we obtain the expansion Eq. (3.9).

Secondly, if we let the operator $D^{k-1}$ act on the Eq. (3.7), then

$$
\begin{aligned}
D^{k-1} f(\mathbf{x}) & =D^{(k-1)}\left(\sum_{i=0}^{k-1}\left(\mathbf{x}^{i} f_{i}(\mathbf{x})\right)\right. \\
& =D^{(k-1)}\left(\left(\mathbf{x}^{k-1} f_{k-1}(\mathbf{x})\right)\right. \\
& =(-1)^{k-1} 2^{k-1}\left[\frac{k-1}{2}\right] ! \mathbb{U}_{\frac{M}{2}} \cdots \mathbb{U}_{\frac{M}{2}+\left[\frac{k-1}{2}\right]-1} f_{k-1}(\mathbf{x}) .
\end{aligned}
$$

By Lemma 3.7, we have

$$
f_{k-1}(\mathbf{x})=\frac{(-1)^{k-1}}{2^{k-1}\left[\frac{k-1}{2}\right] !} J_{\frac{M}{2}+\left[\frac{k-1}{2}\right]-1} \cdots J_{\frac{M}{2}} D^{(k-1)} f(\mathbf{x}) .
$$

Similarly, we let the operator $D^{(k-2)}$ act on $f(\mathbf{x})-\mathbf{x}^{(k-1)} f_{k-1}(\mathbf{x})$, we obtain

$$
\begin{aligned}
D^{(k-2)} & {\left[f(\mathbf{x})-\mathbf{x}^{(k-1)} f_{k-1}(\mathbf{x})\right] } \\
& =D^{(k-2)}\left(\sum_{i=0}^{k-2} \mathbf{x}^{i} f_{i}(\mathbf{x})\right) \\
& =D^{(k-2)}\left(\mathrm{x}^{k-2} f_{k-2}(\mathbf{x})\right) \\
& =(-1)^{k-2} 2^{k-2}\left[\frac{k-2}{2}\right] ! \mathbb{U}_{\frac{M}{2}} \cdots \mathbb{U}_{\frac{M}{2}+\left[\frac{k-2}{2}\right]-1} f_{k-2}(\mathbf{x}) .
\end{aligned}
$$


From Lemma 3.7, we have

$$
f_{k-2}(\mathbf{x})=\frac{(-1)^{k-2}}{2^{k-2}\left[\frac{k-2}{2}\right] !} J_{\frac{M}{2}+\left[\frac{k-2}{2}\right]-1} \cdots J_{\frac{M}{2}} D^{(k-2)}\left[f(\mathbf{x})-\mathbf{x}^{(k-1)} f_{k-1}(\mathbf{x})\right] .
$$

By induction, we have (3.8).

Conversely, suppose that the functions $f_{1}, \cdots, f_{k-1}$ are super monogenic. Using (3.9) and Lemma 3.4, we have

$$
D^{k} f(\mathbf{x})=D^{k}\left[\sum_{i=0}^{k-1} \mathbf{x}^{i} f_{i}(\mathbf{x})\right]=0
$$

which means that the function $f(\mathbf{x})$ given by (3.7) is $k$-super monogenic.

\section{An Expansion for the Operator $D_{\lambda}$}

Definition 4.1. We define the generalized super-Dirac operator by

$$
D_{\lambda}=\partial_{\mathbf{x}}-\lambda,
$$

where $\partial_{\mathbf{x}}$ is the super Dirac operator and $\lambda$ is a complex number.

Denote $\operatorname{ker} D_{\lambda}^{k}=\left\{f \mid(D-\lambda)^{k} f=0, \quad f \in C^{k}(\Omega) \otimes \Lambda_{2 n} \otimes \mathbb{S}_{m \mid 2 n}, k \in \mathbf{N}\right\}$.

Lemma 4.2. If $f \in \operatorname{ker}\left(D_{\lambda}\right)$, then

$$
C_{k} D_{\lambda}^{k} \mathbb{U}_{\lambda}^{k} f=f
$$

where $C_{k}=\frac{1}{k ! \lambda^{k}}$ and $k \in \mathbf{N}$.

Proof. Note that $f \in \operatorname{ker}\left(D_{\lambda}\right)$. For $k=1$, by Lemma 3.4, we observe that

$$
\begin{aligned}
D_{\lambda} \mathbb{U}_{\lambda} f & =\left(\partial_{\mathbf{x}}-\lambda\right) \mathbb{U}_{\lambda} f \\
& =\partial_{\mathbf{x}} \mathbb{U}_{\lambda} f-\lambda \mathbb{U}_{\lambda} f \\
& =\mathbb{U}_{\lambda+1} \partial_{\mathbf{x}} f-\lambda \mathbb{U}_{\lambda} f \\
& =\mathbb{U}_{\lambda+1} \partial_{\mathbf{x}} f-\lambda \mathbb{U}_{\lambda+1} f+\lambda f \\
& =\mathbb{U}_{\lambda+1}\left(\partial_{\mathbf{x}}-\lambda\right) f+\lambda f \\
& =\lambda f .
\end{aligned}
$$

Suppose that for $k=l$,

$$
C_{l} D_{\lambda}^{l} \mathbb{U}_{\lambda}^{l} f=f,
$$

where $C_{l}=\frac{1}{l ! \lambda^{l}}$. For $k=l+1$,

$$
D_{\lambda}^{l+1} \mathbb{U}_{\lambda}^{l} f=D_{\lambda} D_{\lambda}^{l} \mathbb{U}_{\lambda}^{l} f=\frac{1}{C_{l}} D_{\lambda} f=0 .
$$

We calculate

$$
\begin{aligned}
D_{\lambda}^{l+1} \mathbb{U}_{\lambda}^{l+1} f & =D_{\lambda}^{l} D_{\lambda} \mathbb{U}_{\lambda} \mathbb{U}_{\lambda}^{l} f \\
& =D_{\lambda}^{l}\left(\mathbb{U}_{\lambda+1} D_{\lambda}+\lambda\right) \mathbb{U}_{\lambda}^{l} f \\
& =D_{\lambda}^{l} \mathbb{U}_{\lambda+1} D_{\lambda} \mathbb{U}_{\lambda}^{l} f+\lambda D_{\lambda}^{l} \mathbb{U}_{\lambda}^{l} f \\
& =D_{\lambda}^{l-1} D_{\lambda} \mathbb{U}_{\lambda+1} D_{\lambda} \mathbb{U}_{\lambda}^{l} f+\frac{\lambda}{C_{l}} f
\end{aligned}
$$




$$
\begin{aligned}
& =D_{\lambda}^{l-1} \mathbb{U}_{\lambda+2} D_{\lambda}^{2} \mathbb{U}_{\lambda}^{l} f+\frac{2 \lambda}{C_{l}} f \\
& =\cdots \\
& =\mathbb{U}_{\lambda+l+1} D_{\lambda}^{l+1} \mathbb{U}_{\lambda}^{l} f+\frac{(l+1) \lambda}{C_{l}} f \\
& =\frac{1}{C_{l+1}} f .
\end{aligned}
$$

Thus, we have the conclusion.

Theorem 4.3. If $f(x) \in k e r D_{\lambda}^{k}$, then there exist unique functions $f_{0}, \ldots$, $f_{k-1} \in \operatorname{ker} D_{\lambda}$ such that

$$
f(\mathbf{x})=f_{0}(\mathbf{x})+\mathbb{U}_{\lambda} f_{1}(\mathbf{x})+\mathbb{U}_{\lambda}^{2} f_{2}(\mathbf{x})+\cdots+\mathbb{U}_{\lambda}^{k-1} f_{k-1}(\mathbf{x}),
$$

where $f_{0}, \ldots, f_{k-1}$ are given as follows:

$$
\left\{\begin{array}{l}
f_{0}(\mathbf{x})=\left(\mathbf{I}-C_{1} \mathbb{U}_{\lambda} D_{\lambda}\right)\left(\mathbf{I}-C_{2} \mathbb{U}_{\lambda}^{2} D_{\lambda}^{2}\right) \cdots\left(\mathbf{I}-C_{k-1} \mathbb{U}_{\lambda}^{k-1} D_{\lambda}^{k-1}\right) f(\mathbf{x}), \\
f_{1}(\mathbf{x})=C_{1} D_{\lambda}\left(\mathbf{I}-C_{2} \mathbb{U}_{\lambda}^{2} D_{\lambda}^{2}\right) \cdots\left(\mathbf{I}-C_{k-1} \mathbb{U}_{\lambda}^{k-1} D_{\lambda}^{k-1}\right) f(\mathbf{x}), \\
\vdots \\
f_{k-2}(\mathbf{x})=C_{k-2} D_{\lambda}^{k-2}\left(\mathbf{I}-C_{k-1} \mathbb{U}_{\lambda}^{k-1} D_{\lambda}^{k-1}\right) f(\mathbf{x}), \\
f_{k-1}(\mathbf{x})=C_{k-1} D_{\lambda}^{k-1} f(\mathbf{x}),
\end{array}\right.
$$

and $C_{k}=\frac{1}{k ! \lambda^{k}}$.

Conversely, if functions $f_{0}, \ldots, f_{k-1} \in k e r D_{\lambda}$, then the function $f(\mathbf{x})$ given by (4.3) satisfies the equation $D_{\lambda}^{k} f=0$.

Proof. If we let the operator $D_{\lambda}^{k-1}$ act on the Eq. (4.2), then by Lemma 4.2, we have

$$
\begin{aligned}
D_{\lambda}^{k-1} f(\mathbf{x}) & =D_{\lambda}^{k-1}\left(f_{0}(\mathbf{x})+\sum_{i=1}^{k-1}\left(\mathbb{U}_{\lambda}\right)^{i} f_{i}(\mathbf{x})\right) \\
& =D_{\lambda}^{k-1} \mathbb{U}_{\lambda}^{k-1} f_{k-1}(\mathbf{x}) \\
& =\frac{1}{C_{k-1}} f_{k-1}(\mathbf{x}) .
\end{aligned}
$$

Thus,

$$
f_{k-1}(\mathbf{x})=C_{k-1} D_{\lambda}^{k-1} f(\mathbf{x}) .
$$

Similarly, if we let the operator $D_{\lambda}^{k-2}$ act on $f(x)-\mathbb{U}_{\lambda}^{k-1} f_{k-1}(\mathbf{x})$, we have

$$
\begin{aligned}
& D_{\lambda}^{k-2}\left[f(\mathbf{x})-\mathbb{U}_{\lambda}^{k-1} f_{k-1}(\mathbf{x})\right] \\
& =D_{\lambda}^{k-2}\left(f_{0}(\mathbf{x})+\sum_{i=1}^{k-2} \mathbb{U}_{\lambda}^{i} f_{i}(\mathbf{x})\right) \\
& =D_{\lambda}^{k-2} \mathbb{U}_{\lambda}^{k-2} f_{k-2}(\mathbf{x}) \\
& =\frac{1}{C_{k-2}} f_{k-2}(\mathbf{x}) .
\end{aligned}
$$


So

$$
f_{k-2}(\mathbf{x})=C_{k-2} D_{\lambda}^{k-2}\left(\mathbf{I}-C_{k-1} \mathbb{U}_{\lambda}^{k-1} D_{\lambda}^{k-1}\right) f(\mathbf{x}) .
$$

By induction, we have (4.3).

Conversely, suppose that the functions $f_{0}, \ldots, f_{k-1} \in \operatorname{ker} D_{\lambda}$. Applying Lemma 4.2, we obtain

$$
D_{\lambda}^{k} f(\mathbf{x})=D_{\lambda}^{k}\left[f_{0}(\mathbf{x})+\sum_{i=1}^{k-1}\left(\mathbb{U}_{\lambda}\right)^{i} f_{i}(\mathbf{x})\right]=0,
$$

which completes the proof.

\section{Expansions for the Operator $P(D)$}

Let the polynomial

$$
P(\lambda)=\lambda^{k}+b_{0} \lambda^{k-1}+\cdots+b_{k-1},
$$

with $b_{l} \in \mathbb{C}$, and $l=0, \ldots, k-1$. Then the polynomial Dirac operator in super spinor space is defined as

$$
P(D)=D^{k}+b_{0} D^{k-1}+\cdots+b_{k-1} .
$$

Denote ker $P(D)=\left\{f \mid P(D) f=0, f \in C^{k}(\Omega) \otimes \Lambda_{2 n} \otimes \mathbb{S}_{m \mid 2 n}\right\}$.

If $P(\lambda)$ has the decomposition

$$
P(\lambda)=\left(\lambda-\lambda_{0}\right)^{n_{0}} \cdots\left(\lambda-\lambda_{l-1}\right)^{n_{l-1}},
$$

where $\lambda_{i} \in \mathbb{C}$, and $\lambda_{i} \neq 0, i=0, \ldots, l-1$, then the operator $P(D)$ has the decomposition

$$
P(D)=\left(D-\lambda_{0}\right)^{n_{0}} \cdots\left(D-\lambda_{l-1}\right)^{n_{l-1}} .
$$

Lemma 5.1. [10] Let $\pi(\lambda)=\prod_{k=0}^{l-1}\left(\lambda-\lambda_{k}\right)^{n_{k}}$ be a polynomial of $\lambda$, with $\lambda_{k} \in \mathbb{C}, n_{k} \in \mathbf{N}$, and $n_{0}+\cdots+n_{l-1}=s$. Then

$$
\frac{1}{\pi(\lambda)}=\sum_{k=0}^{l-1} \sum_{j=1}^{n_{k}} \frac{1}{\left(n_{k}-j\right) !}\left[\frac{d^{n_{k}-j}}{d \lambda^{n_{k}-j}} \frac{\left(\lambda-\lambda_{k}\right)^{n_{k}}}{\pi(\lambda)}\right]_{\lambda=\lambda_{k}} \frac{1}{\left(\lambda-\lambda_{k}\right)^{j}} .
$$

Lemma 5.2. If $P(D)$ in (5.2) has the decomposition (5.4), then

$$
\operatorname{ker} P(D)=\operatorname{ker} D_{\lambda_{0}}^{n_{0}} \oplus \cdots \oplus \operatorname{ker} D_{\lambda_{l-1}}^{n_{l-1}},
$$

where $\operatorname{ker} D_{\lambda_{i}}^{n_{i}}=\left\{f \mid\left(D-\lambda_{i}\right)^{n_{i}} f=0, \quad f \in C^{n_{i}}(\Omega) \otimes \Lambda_{2 n} \otimes \mathbb{S}_{m \mid 2 n}\right\}$.

Proof. By Lemma 5.1, we have

$$
\operatorname{ker} P(D)=\operatorname{ker} D_{\lambda_{0}}^{n_{0}}+\cdots+\operatorname{ker} D_{\lambda_{l-1}}^{n_{l-1}},
$$

inspired by Gong [10].

Then it is easy to prove that

$$
\operatorname{ker} P(D)=\operatorname{ker} D_{\lambda_{0}}^{n_{0}} \oplus \cdots \oplus \operatorname{ker} D_{\lambda_{l-1}}^{n_{l-1}}
$$

by the division algorithm. 
Theorem 5.3. If $f(\mathbf{x}) \in \operatorname{kerP}(D)$, then there exist unique functions $f_{i, j} \in$ $k e r D_{\lambda_{i}}, i=0, \ldots, l-1, j=0, \ldots, n_{i}-1$, such that

$$
f=\sum_{i=0}^{l-1} f_{i, 0}+\sum_{i=0}^{l-1} \sum_{j=1}^{n_{i}-1} \mathbb{U}_{\lambda}^{j} f_{i, j}
$$

where $f_{i, 0}, \ldots, f_{i, n_{i}-1}$ are given as follows:

$$
\begin{aligned}
& \left\{\begin{array}{l}
f_{i, 0}(\mathbf{x})=\left(\mathbf{I}-C_{1} \mathbb{U}_{\lambda} D_{\lambda}\right)\left(\mathbf{I}-C_{2} \mathbb{U}_{\lambda}^{2} D_{\lambda}^{2}\right) \cdots\left(\mathbf{I}-C_{n_{i}-1} \mathbb{U}_{\lambda}^{n_{i}-1} D_{\lambda}^{n_{i}-1}\right) f(\mathbf{x}) \\
f_{i, 1}(\mathbf{x})=C_{1} D_{\lambda}\left(\mathbf{I}-C_{2} \mathbb{U}_{\lambda}^{2} D_{\lambda}^{2}\right) \cdots\left(\mathbf{I}-C_{n_{i}-1} \mathbb{U}_{\lambda}^{n_{i}-1} D_{\lambda}^{n_{i}-1}\right) f(\mathbf{x}) \\
\quad \vdots \\
f_{i, n_{i}-2}(\mathbf{x})=C_{n_{i}-2} D_{\lambda}^{n_{i}-2}\left(\mathbf{I}-C_{n_{i}-1} \mathbb{U}_{\lambda}^{n_{i}-1} D_{\lambda}^{n_{i}-1}\right) f(\mathbf{x}) \\
f_{i, n_{i}-1}(\mathbf{x})=C_{n_{i}-1} D_{\lambda}^{n_{i}-1} f(\mathbf{x})
\end{array}\right. \\
& \text { and } C_{k}=\frac{1}{k ! \lambda^{k}}
\end{aligned}
$$

Proof. Note that $P(D) f=0$. It follows by Lemma 5.2 that there exist unique functions $f_{i}, i=0, \ldots, l-1$ such that

$$
f=f_{0}+f_{1}+\cdots+f_{l-1}
$$

where $f_{i} \in \operatorname{ker} D_{\lambda_{i}}^{n_{i}}$.

Theorem 4.3 implies that there exist unique functions $f_{i, j}, i=0, \ldots, l-$ $1, j=1, \ldots, n_{i}-1$, such that

$$
f_{i}=f_{i, 0}+\sum_{j=1}^{n_{i}-1} \mathbb{U}_{\lambda}^{j} f_{i, j},
$$

where $f_{i, j}$ are given in Theorem 4.3 .

Then the proof is completed.

If $P(\lambda)$ has the decomposition

$$
P(\lambda)=(\lambda-0)^{n_{0}}\left(\lambda-\lambda_{1}\right)^{n_{1}} \cdots\left(\lambda-\lambda_{k}\right)^{n_{k}},
$$

where $\lambda_{i} \in \mathbb{C}$, and $\lambda_{i} \neq 0, i=1, \ldots, k$, the polynomial Dirac operator in super spinor space $P(D)$ has the decomposition

$$
P(D)=D^{n_{0}}\left(D-\lambda_{1}\right)^{n_{1}} \cdots\left(D-\lambda_{k}\right)^{n_{k}} .
$$

Applying Theorems 4.3 and 5.3, we obtain the following theorem:

Theorem 5.4. If $f(\mathbf{x}) \in \operatorname{kerP}(D)$, then there exist unique functions $f_{0, l} \in$ $\operatorname{ker} D, l=1, \ldots, n_{0}$, and $f_{i, j} \in \operatorname{ker} D_{\lambda_{i}}, i=1, \ldots, k, j=0, \ldots, n_{i}-1$ such that

$$
f=\sum_{l=1}^{n_{0}} \mathbf{x}^{l-1} f_{0, l}+\sum_{i=1}^{k} f_{i, 0}+\sum_{i=1}^{k} \sum_{j=1}^{n_{i}-1} \mathbb{U}_{\lambda}^{j} f_{i, j}
$$


where $f_{i, 0}, \ldots, f_{i, n_{i}-1}$ are given as follows:

$$
\left\{\begin{array}{l}
f_{i, 0}(\mathbf{x})=\left(\mathbf{I}-C_{1} \mathbb{U}_{\lambda} D_{\lambda}\right)\left(\mathbf{I}-C_{2} \mathbb{U}_{\lambda}^{2} D_{\lambda}^{2}\right) \cdots\left(\mathbf{I}-C_{n_{i}-1} \mathbb{U}_{\lambda}^{n_{i}-1} D_{\lambda}^{n_{i}-1}\right) f(\mathbf{x}), \\
f_{i, 1}(\mathbf{x})=C_{1} D_{\lambda}\left(\mathbf{I}-C_{2} \mathbb{U}_{\lambda}^{2} D_{\lambda}^{2}\right) \cdots\left(\mathbf{I}-C_{n_{i}-1} \mathbb{U}_{\lambda}^{n_{i}-1} D_{\lambda}^{n_{i}-1}\right) f(\mathbf{x}) \\
\quad \vdots \\
f_{i, n_{i}-2}(\mathbf{x})=C_{n_{i}-2} D_{\lambda}^{n_{i}-2}\left(\mathbf{I}-C_{n_{i}-1} \mathbb{U}_{\lambda}^{n_{i}-1} D_{\lambda}^{n_{i}-1}\right) f(\mathbf{x}) \\
f_{i, n_{i}-1}(\mathbf{x})=C_{n_{i}-1} D_{\lambda}^{n_{i}-1} f(\mathbf{x})
\end{array}\right.
$$

with $C_{k}=\frac{1}{k ! \lambda^{k}}$, and $f_{0, l}, l=1, \ldots, n_{0}$ are given as follows:

$$
\left\{\begin{array}{l}
f_{0,1}(\mathbf{x})=f(\mathbf{x})-\mathbf{x}^{n_{0}-1} f_{n_{0}-1}(\mathbf{x})-\cdots-\mathbf{x} f_{0,2}(\mathbf{x}) \\
f_{0,2}(\mathbf{x})=\frac{-1}{2} J_{\frac{M}{2}} D\left[f(\mathbf{x})-\mathbf{x}^{n_{0}-1} f_{0, n_{0}-1}(\mathbf{x})-\cdots-\mathbf{x}^{2} f_{0,3}(\mathbf{x})\right] \\
\quad \vdots \\
f_{0, n_{0}-1}(\mathbf{x}) \\
\quad=\frac{(-1)^{n_{0}-1}}{2^{n_{0}-1}\left[\frac{n_{0}-1}{2}\right] !} J_{\frac{M}{2}+\left[\frac{n_{0}-1}{2}\right]-1} \cdots J_{\frac{M}{2}} D^{n_{0}-2}\left[f(\mathbf{x})-\mathbf{x}^{\left(n_{0}-1\right)} f_{0, n_{0}}(\mathbf{x})\right] \\
f_{0, n_{0}}(\mathbf{x})=\frac{(-1)^{n_{0}}}{2^{n_{0}}\left[\frac{n_{0}}{2}\right] !} J_{\frac{M}{2}+\left[\frac{n_{0}}{2}\right]-1} \cdots J_{\frac{M}{2}} D^{n_{0}-1} f(\mathbf{x}),
\end{array}\right.
$$

\section{Generalized Riquier Problem in Super Spinor Space}

In this section, we investigate the generalized Riquier problem in super spinor space by an expansion for the operator $D_{\lambda}^{k}$, as follows:

Given $g_{i}(\mathbf{y}) \in C(\partial \Omega) \otimes \Lambda_{2 n} \otimes \mathbb{S}_{m \mid 2 n}$, find a function $f$ such that $D_{\lambda}^{i} f \in$ $C(\bar{\Omega}) \otimes \Lambda_{2 n} \otimes \mathbb{S}_{m \mid 2 n}$ for $i=0, \cdots, k-1$, and

$$
\left\{\begin{array}{l}
D_{\lambda}^{k} f=0, \quad f \in C^{k}(\Omega) \otimes \Lambda_{2 n} \otimes \mathbb{S}_{m \mid 2 n}, \\
\left.D_{\lambda}^{i} f\right|_{\partial \Omega}=g_{i}(\mathbf{y}) .
\end{array}\right.
$$

Theorem 6.1. Suppose that $f_{i}(\mathbf{x}), i=0, \cdots, k-1$, satisfy the following equations

$$
\left\{\begin{array}{l}
D_{\lambda} f_{i}(\mathbf{x})=0, \quad f_{i}(\mathbf{x}) \in C^{2 i+1}(\Omega) \otimes \Lambda_{2 n} \otimes \mathbb{S}_{m \mid 2 n} \\
\left.f_{i}(\mathbf{x})\right|_{\partial \Omega}=\frac{1}{i ! \lambda^{i}}\left[g_{i}(\mathbf{y})-\left.\sum_{j=i+1}^{k-1} D_{\lambda}^{i} \mathbb{U}_{\lambda}^{j} f_{j}(\mathbf{x})\right|_{\partial \Omega}\right], i=0, \cdots, k-2 \\
f_{i}(\mathbf{x}) \in C(\bar{\Omega}) \otimes \Lambda_{2 n} \otimes \mathbb{S}_{m \mid 2 n}, \quad D_{\lambda}^{i} \mathbb{U}_{\lambda}^{j} f_{j}(\mathbf{x}) \in C(\bar{\Omega}) \otimes \Lambda_{2 n} \otimes \mathbb{S}_{m \mid 2 n} \\
\left.f_{k-1}(\mathbf{x})\right|_{\partial \Omega}=\frac{1}{(k-1) ! \lambda^{k-1}} g_{k-1}(\mathbf{y}), \quad f_{k-1}(\mathbf{x}) \in C(\bar{\Omega}) \otimes \Lambda_{2 n} \otimes \mathbb{S}_{m \mid 2 n}
\end{array}\right.
$$

Then the function $f(\mathbf{x})$ given by

$$
f(\mathbf{x})=\sum_{i=0}^{k-1} \mathbb{U}_{\lambda}^{i} f_{i}(\mathbf{x})=f_{0}(\mathbf{x})+\sum_{i=1}^{k-1} \mathbb{U}_{\lambda}^{i} f_{i}(\mathbf{x}),
$$

is a solution of the problem (6.1). 
Proof. Let $f_{i}(\mathbf{x}) \in C^{2 i+1}(\Omega) \otimes \Lambda_{2 n} \otimes \mathbb{S}_{m \mid 2 n}, i=0, \cdots, k-1$. Because the functions $f_{i}(\mathbf{x})$ satisfy the equations $D_{\lambda} f_{i}(\mathbf{x})=0$, it follows by Theorem 4.3 that

$$
D_{\lambda}^{k} f(\mathbf{x})=0,
$$

where $f(\mathbf{x})$ is given in (6.3). For $0 \leq i<k-1$, Lemma 4.2 implies that

$$
\begin{aligned}
D_{\lambda}^{i} f(\mathbf{x}) & =D_{\lambda}^{i}\left(f_{0}(\mathbf{x})+\sum_{j=1}^{k-1} \mathbb{U}_{\lambda}^{j} f_{j}(\mathbf{x})\right) \\
& =i ! \lambda^{i} f_{i}(\mathbf{x})+\sum_{j=i+1}^{k-1} D_{\lambda}^{i} \mathbb{U}_{\lambda}^{j} f_{j}(\mathbf{x}) .
\end{aligned}
$$

Note that $f_{i}(\mathbf{x}) \in C(\bar{\Omega}) \otimes \Lambda_{2 n} \otimes \mathbb{S}_{m \mid 2 n}$ and $D_{\lambda}^{i} \mathbb{U}_{\lambda}^{j} f_{j}(\mathbf{x}) \in C(\bar{\Omega}) \otimes \Lambda_{2 n} \otimes \mathbb{S}_{m \mid 2 n}$. Then $D_{\lambda}^{i} f(\mathbf{x}) \in C(\bar{\Omega}) \otimes \Lambda_{2 n} \otimes \mathbb{S}_{m \mid 2 n}$.

For $i=k-1$,

$$
D_{\lambda}^{k-1} f(\mathbf{x})=D_{\lambda}^{k-1}\left(f_{0}(\mathbf{x})+\sum_{j=1}^{k-1} \mathbb{U}_{\lambda}^{j} f_{j}(\mathbf{x})\right)=(k-1) ! \lambda^{k-1} f_{k-1}(\mathbf{x}) .
$$

Because $f_{k-1}(\mathbf{x}) \in C(\bar{\Omega}) \otimes \Lambda_{2 n} \otimes \mathbb{S}_{m \mid 2 n}$, it follows that $D_{\lambda}^{k-1} f(\mathbf{x}) \in$ $C(\bar{\Omega}) \otimes \Lambda_{2 n} \otimes \mathbb{S}_{m \mid 2 n}$

Letting $\mathbf{x} \rightarrow \partial \Omega$, and using the second equality and the third equality in (6.2), we have

$$
\left.D_{\lambda}^{i} f\right|_{\partial \Omega}=g_{i}(\mathbf{y}), i=0, \ldots, k-1 .
$$

Thus, we have the conclusion.

\section{Acknowledgments}

This research was supported by the TianYuan Special Funds of the National Natural Science Foundation of China under Grant No. 11426082.

Open Access. This article is distributed under the terms of the Creative Commons Attribution 4.0 International License (http://creativecommons.org/licenses/ by/4.0/), which permits unrestricted use, distribution, and reproduction in any medium, provided you give appropriate credit to the original author(s) and the source, provide a link to the Creative Commons license, and indicate if changes were made.

\section{References}

[1] Almansi, E.: Sull'integrazione dell'equazione differenziale $\Delta^{2 m} u=0$. Ann. Math. Pura. Appl. 2(3), 1-51 (1898)

[2] Altin, A.: Some expansion formulas for a class of singular partial differential equations. Proc. Am. Mat. Soc. 85(1), 42-46 (1982) 
[3] Aronszajn, N., Creese, T.M., Lipkin, L.J.: Polyharmonic Functions. Oxford Mathematics Monographs, The Clarendon Press, Oxford University Press, New York (1983)

[4] Bu, Y., Du, J.Y.: The RH boundary value problem of the k-monogenic functions. J. Math. Anal. Appl. 347, 633-644 (2008)

[5] Coulembier, K.: On a class of tensor product representations for the orthosymplectic superalgebra. J. Pure Appl. Algebra. 217, 819-837 (2013)

[6] Coulembier, K.: The orthosymplectic superalgebra in harmonic analysis. J. Lie Theory 23, 55-83 (2013)

[7] Coulembier, K., De Bie, H.: Conformal symmetries of the super Dirac operator. Rev. Mat. Iberoam. 31(2), 373-410 (2015)

[8] Delanghe, R., Sommen, F., Soucek, V.: Clifford Algebra and Spinor-Valued Functions, vol. 53 of Mathematics and its Applications. Kluwer Academic Publishers Group, Dordrecht (1992)

[9] Fegan, H.: Conformally invariant first order differential operators. Quart. J. Math. Oxford. 2(27), 371-378 (1976)

[10] Gong, Y.F., Qian, T, Du, J.Y: Structure of solutions of polynomial Dirac equations in Clifford analysis. Complex Var. 49(1), 15-24 (2003)

[11] Hedenmalm, H.: A computation of Green functions for the weighted biharmonic operators $\Delta|z|^{-2 \alpha}$, with $\alpha>1$. Duke Math. J. 75, 51-78 (1994)

[12] Kostant, B.: Symplectic Spinors. In: Symposia Mathematica vol. XIV, pp. 139152. Academic Press, Amsterdam (1974)

[13] Krýsl, S.: Howe type duality for metaplectic group acting on symplectic spinor valued forms. J. Lie Theory. 22, 1049-1063 (2012)

[14] Malonek, H., Ren, G.B.: Almansi-type theorems in Clifford analysis. Math. Meth. Appl. Sci. 25, 1541-1552 (2002)

[15] Özalp, N., Çetinkaya, A.: Expansion formulas and Kelvin principle for a class of partial differential equations. Math. Balkanica New Ser. 15, 220-226 (2001)

[16] Pavlovic, M.: Decompositions of $L_{p}$ and Hardy spaces of polyharmonic functions. J. Math. Anal. Appl. 216, 499-509 (1997)

[17] Qiao, Y.Y., Yuan, H.F., Yang, H.J.: Normalized system for the super Laplace operator. Adv. Appl. Clifford Alg. 22(4), 1109-1128 (2012)

[18] Ryan, J.: Basic Clifford analysis. Cubo Math. Educ. 2, 226-256 (2000)

[19] Stein, E., Weiss, G.: Generalization of the Cauchy-Riemann equations and representations of the rotation group. Am. J. Math. 90, 163-196 (1968)

[20] Yuan, H.F., Qiao, Y.Y.: Solutions of the Dirac and related equations in superspace. Complex Var. Elliptic Equ. 59(9), 1315-1327 (2014)

[21] Yuan, H.F., Qiao, Y.Y., Yang, H.J.: Decomposition of k-monogenic functions in superspace. Complex Var. Elliptic Equ. 58(8), 1109-1124 (2013)

[22] Yuan, H.F., Zhang, Z.H., Qiao, Y.Y.: Polynomial Dirac operators in superspace. Adv. Appl. Clifford Algebras. 25(3), 755-769 (2015) 


\author{
Hongfen Yuan \\ College of Science \\ Hebei University of Engineering \\ Handan 056038 \\ People's Republic of China \\ e-mail: yhf0609@163.com
}

Received: February 07, 2015.

Accepted: September 02, 2015. 